\title{
A new model for retrieving information about turbulence intensity from radar signal
}

\author{
Felix J. Yanovsky, Daria M. Turenko \\ National Aviation University, Kiev, Ukraine \\ yanovsky@nau.edu.ua
}

\author{
A.C.P. Oude Nijhuis, O.A. Krasnov, and A. Yarovoy \\ TU-Delft, the Netherlands \\ albertoudenijhuis@gmail.com
}

\begin{abstract}
This paper presents mathematical models that are necessary to describe the relationship between Doppler spectrum width of the signal reflected from precipitation and eddy dissipation rate of turbulence kinetic energy. The feature of the models is taking into account the contribution of drop fall velocity variance, which is analyzed in detail, and the inertia of drops. The calculations are done for different turbulence intensity and drop size distribution. The model is suitable for substantiation of allowable errors at EDR estimation and radar signal processing to retrieve information about turbulence intensity.
\end{abstract}

Keywords—remote sensing; meteorological radar; turbulence intensity retrieval; Doppler spectrum

\section{INTRODUCTION}

Meteorological conditions and weather hazards influence significantly to flight safety and fulfilment of aviation operations. Strong and even moderate turbulent zones should be avoided at all stages of flight. Due to increasing intensity of flights, it is important to minimize the time between possible pairs of landing and/or taking off. From this point of view, dangerous wake-vortex became one of the most important limiting factors of airport capacity. It means that it is necessary to have a tool for forecasting the time of wake-vortex life. While strong turbulence is dangerous itself, some light turbulence may play a positive role because it helps to dispersal process of the wake-vortex created by the aircraft during landing.

That means that even light turbulence is necessary to be measured. Result of such measurement can be used to predict the duration of dangerous wake-vortexes, which are among the most important limiting factors of airport capacity. In order to estimate atmosphere turbulence quantitatively, the ICAO recommends using the Eddy Dissipation Rate (EDR).Turbulence $\operatorname{EDR}(\varepsilon)$ is one of the fundamental parameters that describes turbulence characteristics. It represents the rate of energy cascading larger eddies to smaller ones until the energy is transformed to heat. Knowledge of the EDR in troposphere is also important in understanding the thermal and dynamical structures as well as studying the mixing of minor constituents. EDR is also one of the key parameters in numerical models of the atmosphere.

EDR can be retrieved from measurements of in-situ sonic anemometers, and also with help of balloons and aircraft. However a great advantage of modern remote tools like radar and lidar is the possibility to observe large volumes of the atmosphere with rather high resolution. There are a lot of works on estimation of EDR based on radar remote sensing data. Many of radar methods and signal processing algorithms are based on estimating Doppler spectrum width (DSW) [1-5]. It is known that the DSW provides an overestimate of the turbulence EDR because not only turbulence contributes to the width of the spectrum. Different corrections were proposed, usually, dependent on the features of scatterers and conditions of observation. In turn, measurable radar variables like DSW should be accurately measured in operational radar systems. This means that signal processing algorithms should be suitable for processing limited and even rather short samples of radar signals [5]. Adaptive signal processing method to correct measured DSW before its transformation into EDR value [6] includes estimation of nonturbulent contribution into DSW using measurable parameters and an algorithm for adaptive correction. In case of slant and especially vertical sounding the most important nonturbulent component, which contributes to DSW, is the variance of scatterer fall velocities. The most important type of such scatterers is the class of water drops. The drop fall velocity variance under different conditions and its influence to DSW still is not studied carefully enough.

In this paper we propose mathematical models that allow calculating drop fall velocity and turbulent contributions to Doppler spectrum in order to use this information in an adaptive approach for retrieving EDR from radar signal reflected from clouds and precipitation.

\section{DOPPLER SPECTRUM WIDTH IN A RESOLUTION VOLUME AS AN INFORMATIVE PARAMETER FOR EDR RETRIEVAL}

Doppler power spectra represent the reflectivity-weighted scatterer velocity distribution. If the scatterers are raindrops, the DSW is related to the raindrop fall velocity distribution width and the turbulence spectrum width. For the estimation of turbulence-induced spectral broadening, we use the adaptive approach proposed in [6]. The idea is to apply radar measurements to estimate a contribution caused by the variance of droplet fall velocity first, and then subtract it from the measured Doppler velocity variance. It is assumed that the residual represents the DSW directly related with turbulence. The spatial scales of turbulence that influence to scatterers are limited by a size of the resolution volume in this case. However in case of Kolmogorov isotropic turbulence model, EDR is stable in the framework of the inertial subrange [7]. 
If we assume that the fall speed and turbulent speed are uncorrelated, then we can write the radar Doppler spectral variance as:

$$
\sigma_{v}^{2}=\sigma_{v, f}^{2}+\sigma_{v, T}^{2}
$$

where $\sigma_{v, f}$ is the spectral width due to dispersion of fall velocity, and $\sigma_{v, T}$ is the spectral width due to turbulence. After subtracting $\sigma_{v, f}^{2}$ from (1) we can use the residue as measured estimation $\hat{\sigma}_{v, T}^{2}$ to retrieve the EDR: $\varepsilon \approx f\left(\sigma_{v, T}\right)[8]$.

In practice, estimating $\sigma_{v, f}$ can be done using polarimetric radar measurements because there are several polarimetric parameters, which are very sensitive to the shape of scatterers [9]. In turn, a shape of a raindrop is related to its equivalent diameter. Having information about microstructure of the resolution volume in the form of drop size distribution (DSD) or parameters of $\mathrm{DSD}$, one can calculate $\sigma_{v, f}$, taking into account that terminal drop fall velocity is associated with a size of the drop according to the Stokes law.

However polarimetric approach does not work effectively in case of sounding rain into the zenith because the beam projection of a raindrop becomes a circle, that is, it is not sensitive to polarization. That is why when using radar signals reflected from rain events under the condition that the radar beam was pointing vertically, the signal processing can be made using a simplified method for retrieving DSD parameters according to [6].

Rough estimation of the median drop diameter $D_{0}$ can be made from radar equivalent reflectivity $Z_{H} \approx Z_{V}=Z$ in a given resolution volume. Given well-known empirical expression, which associates measured $Z$ with the rain rate $R$ [8], $Z \approx 200 R^{1.6}$ and moreover, $D_{0} \approx 0.902 R^{21}$, one can use approximate formula to get the $D_{0}[\mathrm{~mm}]$ estimate from measured radar reflectivity $\left[\mathrm{mm}^{6} / \mathrm{m}^{3}\right]$ :

$$
\widehat{D}_{0} \approx 0.447 Z^{0.131} \text {. }
$$

Knowing $\widehat{D}_{0}$ and made a supposition about spread (or shape) parameter $\mu$ (e.g. $\mu=5)$ it is possible to retrieve drop size distribution (DSD) in the form of gamma distribution:

$$
N(D)=N_{0} D^{\mu} \exp (-\Lambda D)=N_{0} D^{\mu} \exp \left(-\frac{3.67+\mu}{D_{0}} D\right)
$$

where $D$ is the drop equivalent diameter, $N(D)$ is the number of drops with an equivalent diameter between $D$ and $D+d D$ in a unit of volume, $\mu$ is the shape parameter, and $N_{0}$ is a scaling factor (drop concentration); $\Lambda$ is a slope parameter, which is related to the median diameter $D_{0}$. The next step is a transition from DSD to the drop fall velocity (DFV) distribution and DSW $\sigma_{v, f}$ due to a dispersion of drop fall velocity.

\section{MATH MODELS OF DROP FALL VELOCITY CONTRIBUTION} TO THE DOPPLER SPECTRUM WIDTH

Dependence of the radial component of the terminal fall velocity of a raindrop along the radar beam upon the equivalent diameter of the drop in accordance with Atlas' approximation [8] and taking into account antenna elevation $\theta$ can be expressed as

$$
v_{f}(D)=\alpha-\beta \exp (-0.6 D), \quad D \geq 0.109 \mathrm{~mm}
$$

with $\alpha=9.65 \sin \theta ; \beta=10.3 \sin \theta$.

Based on DSD model (3) and relationship (4) we can derive the DFV distribution $N_{f}\left(v_{f}\right)$ as a distribution of a function of a random value subjected to the known distribution, using the inverse function $D=f\left(v_{f}\right)$ from (4) and its derivative over $D$. Then

$$
N_{f}\left(v_{f}\right)=N\left(\left[v_{f}(D)\right]^{-1}\right) \frac{d}{d D}\left(\left[v_{f}(D)\right]^{-1}\right) .
$$

After substitution we get

$$
N_{f}\left(v_{f}\right)=\frac{\left(-1.667 \ln \frac{\alpha-v_{f}}{\beta}\right)^{\mu} \exp \left(1.667 \frac{3.67+\mu}{D_{0}} \ln \frac{\alpha-v_{f}}{\beta}\right)}{0.6\left(\alpha-v_{f}\right)\left(\frac{D_{0}}{3.67+\mu}\right)^{\mu+1} \cdot \mu !}
$$

Plots of calculations done by formula (6) are presented in Fig. 1 at three values of $\mu\left(2 ; 5\right.$; and 9), $D_{0}=1.5 \mathrm{~mm}, \theta=90 \mathrm{deg}$ (vertical sounding).

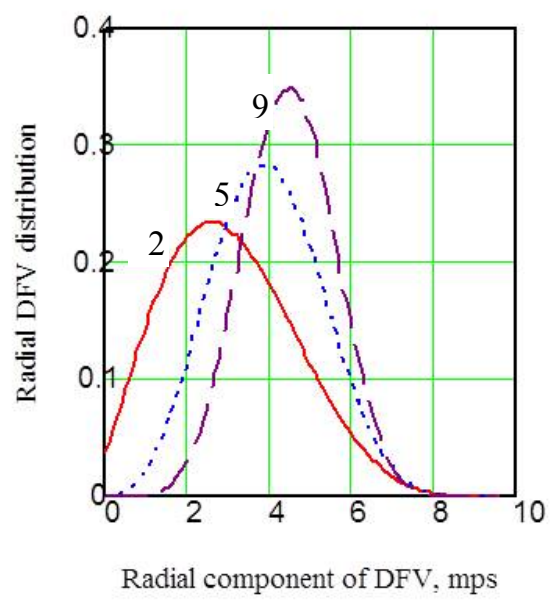

Fig. 1. Radial DFV distribution at different $\mu(2 ; 5 ; 9)$ and vertical vertical sounding.

Having distribution (6), it is easy to calculate its moments of the $n$-th order $\int v_{f}^{n} N_{f}\left(v_{f}\right) d v_{f}, n=0 ; 1 ; 2 ; \ldots$ and the RMS of radial velocity $W_{d}$ due to gravity

$$
W_{d}^{2}=\int v_{f}^{2} N_{f}\left(v_{f}\right) d v_{f}-\left[\int v_{f} N_{f}\left(v_{f}\right) d v_{f}\right]^{2}
$$

that is shown in Fig. 2. 


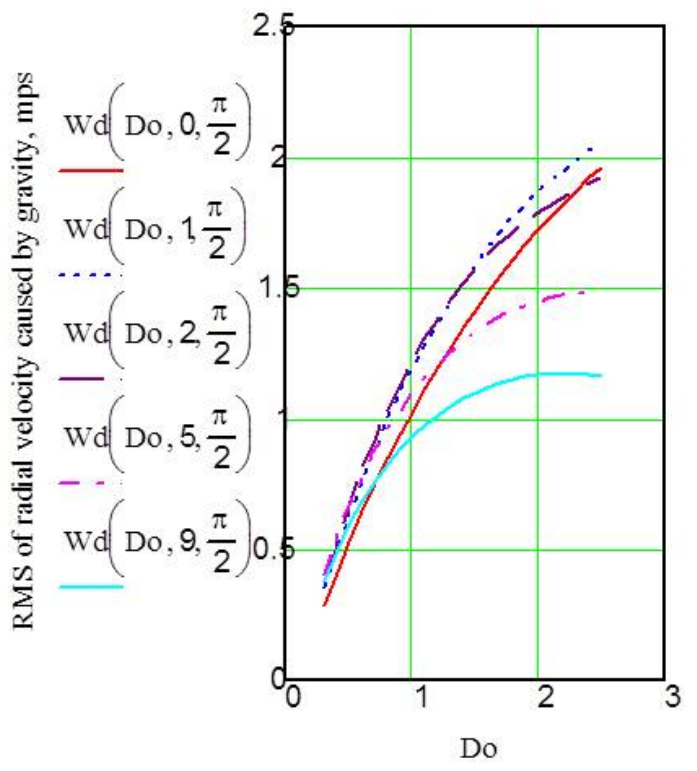

Median drop diameter, $\mathrm{mm}$

Fig. 2. RMS of radial velocity caused by drop fall vs median drop diameter.

The calculated RMS of radial velocity caused by gravity, that is, by dispersion of drop fall, is the basis for a drop fall velocity contribution to the DSW, however it is not a DSW caused by gravity itself. In order to estimate DSW $\sigma_{v, f}$ that contributes to (1), one should take into account that Doppler spectrum is a radial velocity distribution weighted by radar cross section (RCS) of raindrops, which under the condition of $\lambda \gg D$ is proportional to $D^{6}$. Hence we can write down the $1^{\text {st }}$ and $2^{\text {nd }}$ moments, that is, $\overline{v_{f}}$ and $\overline{v_{f}^{2}}$ correspondingly:

$$
\begin{gathered}
\overline{v_{f}}=\frac{\int_{0}^{\infty} v_{f} N_{f}\left(v_{f}\right)\left[D\left(v_{f}\right)\right]^{6} d v_{f}}{\int_{0}^{\infty} N_{f}\left(v_{f}\right)\left[D\left(v_{f}\right)\right]^{6} d v_{f}}, \\
\overline{v_{f}^{2}}=\frac{\int_{0}^{\infty} v_{f}^{2} N_{f}\left(v_{f}\right)\left[D\left(v_{f}\right)\right]^{6} d v_{f}}{\int_{0}^{\infty} N_{f}\left(v_{f}\right)\left[D\left(v_{f}\right)\right]^{6} d v_{f}},
\end{gathered}
$$

where $D\left(v_{f}\right)$ is the inverse function of (4)

$$
D\left(v_{f}\right)=-1.667 \ln \left(\frac{\alpha-v_{f}}{\beta}\right) .
$$

Thus, RMS of Doppler velocity induced by gravity or DFV contribution to the Doppler spectrum width is

$$
W_{d n}=\left[\overline{v_{f}^{2}}-\left(\bar{v}_{f}\right)^{2}\right]^{-2}=\hat{\sigma}_{v, f}
$$

The results of $W_{d n}$ calculations made according to the considered approach are presented in Fig. 3.

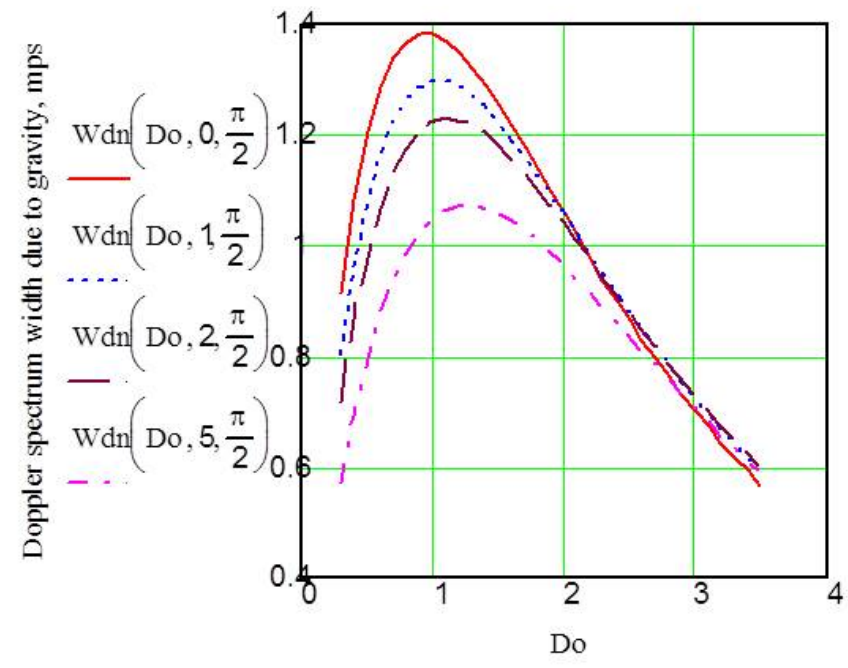

Median drop diameter, $\mathrm{mm}$

Fig. 3. Contribution of DFV to the DSW vs median drop diameter at different shape parameter $\mu$.

Based on the expression (6) it is possible to conduct a useful simulation study of the appropriate processes. Another way to estimate the DFV distribution width weighted by RSC $\sigma(D) \propto D^{6}$ directly from DSD (3) is suitable, if we do not need the full expression of DFV distribution (6). In this case it is enough to calculate the mean DFV as

$$
\overline{v_{f}}=\frac{\int_{0}^{\infty} v_{f}(D) N(D) D^{6} d D}{\int_{0}^{\infty}(D) N(D) D^{6} d D},
$$

and the $2^{\text {nd }}$ moment is

$$
\overline{v_{f}^{2}}=\frac{\int_{0}^{\infty} v_{f}^{2}(D) N(D) D^{6} d D}{\int_{0}^{\infty}(D) N(D) D^{6} d D}
$$

Analytical solutions of (12) and (13) after substitution DSD from (3), derived in [10], are:

$$
\overline{v_{f}}=\alpha-\beta\left[\frac{3.67+\mu}{3.67+\mu+0.6 D_{0}}\right]^{\mu+7}
$$

$\overline{v_{f}^{2}}=\alpha^{2}-2 \alpha \beta\left[\frac{3.67+\mu}{3.67+\mu+0.6 D_{0}}\right]^{\mu+7}+\beta^{2}\left[\frac{3.67+\mu}{3.67+\mu+1.2 D_{0}}\right]^{\mu+7}$

The spectral width due to the dispersion of fall velocities is then again calculated according to (11). The result is completely the same as in case of using expressions (8) and (9) presented in Fig. 3. One can see that the DFV contribution to the DSW has a maximum at some value of $D_{0}$ (and hence at some rain rate) and then decreases in spite of the fact that the 
variance of drop fall velocity distribution increases monotonously (Fig. 2). This is a result of weighting by RCS because the slope of the dependence $v_{f}(D)$ given by (4) decreases significantly for large drops (about more than $3 \mathrm{~mm}$ ) and the influence of the drop diameter is extremely strong $\left(R C S \propto D^{6}\right)$.

\section{TURBULENCE CONTRIBUTION TO THE DOPPLER SPECTRUM WIDTH}

The distribution of turbulence-induced velocity of drops according to [11] can be described by the model

$$
p_{T}\left[v_{T} / L_{t h}(D)\right]=\frac{\left[{ }^{*}\right]}{\pi^{1 / 2} C_{0} L_{o}^{2 / 3} \varepsilon^{2 / 3}},
$$

where symbol [*] means

$\left[\begin{array}{l}2^{1 / 2} \varepsilon^{1 / 3} L_{u l}^{1 / 3} C_{0}^{1 / 2} \exp \left(-\frac{v_{t}^{2}}{2 \varepsilon^{2 / 3} L_{u l}^{2 / 3} C_{0}}\right)-2^{1 / 2} \varepsilon^{1 / 3} L_{t h}^{1 / 3} C_{0}^{1 / 2} \exp \left(-\frac{v_{t}^{2}}{2 \varepsilon^{2 / 3} L_{t h}^{2 / 3} C_{0}}\right)+ \\ \pi^{1 / 2} v \cdot \operatorname{erf}\left(\frac{v_{t}}{2^{1 / 2} \varepsilon^{1 / 3} L_{u l}^{1 / 3} C_{0}^{1 / 2}}\right)-\pi^{1 / 2} v \cdot \operatorname{erf}\left(\frac{v_{t}}{2^{1 / 2} \varepsilon^{1 / 3} L_{t h}^{1 / 3} C_{0}^{1 / 2}}\right)\end{array}\right]$,

where $L_{o}$ is the outer scale of turbulent flow and $L_{u l}$ is max scale that acts in the resolution volume. This model takes into account the inertia of drops [12] that depends on the drop diameter $D$, which is an argument of the function

$$
L_{t h}(D)=21.17\left(1-e^{-0.527 D}\right) V_{w},
$$

where $L_{t h}(D)$ means a threshold scale, that is, a minimal spatial scale of turbulence affecting raindrops with equivolumetric diameter $\leq D[\mathrm{~mm}] ; V_{w}$ is a dimension constant $[\mathrm{m} / \mathrm{s}]$ that relates droplet relaxation time with the scale of turbulence; $L_{t h}(D)$ in (16) and (17) is in meter. Limiting scale $L_{u l}$, which is the maximum spatial scale of turbulence contributing to random motion of scatterers that form the reflected signal, is the largest characteristic size of a single resolution volume in radial or tangential direction. Dimensionless constant $C_{0}$ of unity order came from Kolmogorov turbulence law, and $\varepsilon$ is EDR.

Distribution (16) has zero mean $\bar{v}_{T}=0$. Hence, the Doppler spectral variance caused by turbulence is:

$$
\sigma_{v, T}^{2}=\frac{\int_{0}^{\infty} \int_{-\infty}^{\infty} v_{t}^{2} p_{T}(D) D^{6} N(D) d v_{T} d D}{\int_{0}^{\infty} \int_{-\infty}^{\infty} p_{T}(D) D^{6} N(D) d v_{T} d D}
$$

In case of numerical calculations the limits of integration over $D$ should be taken not from 0 but from $D_{\min } \geq 0.109$ in accordance with (4) to $D_{\max }$ that can be chosen as $8 \mathrm{~mm}$ because larger rain droplets cannot exist. Infinite limits of integration over $v_{T}$ can also be substituted by some robust values that are large enough. The multiplier $D^{6}$ in the integrand provides weighting by RCS similarly to (8), (9), (12), and (13). After the substitution $p_{T}(D)=f\left(D, D_{0}, \mathcal{\varepsilon}, \mu\right)$ from
(16) and $N(D)=f\left(D_{0}, \mu\right)$ from (3) we can calculate the turbulence contribution to the variance of the Doppler spectrum at the sounding of rain. Note that the raindrop concentration $N_{0}$, which is a parameter of the initial DSD (3), is absent in DFV distribution (6), as well as for calculations according to (12), (13), and (18) it is not necessary to know $N_{0}$.

The result of calculating variance $(18)\left[\mathrm{m}^{2} / \mathrm{s}^{2}\right]$ versus EDR $\left[\mathrm{m}^{2} \mathrm{~s}^{-3}\right] \sigma_{v, T}^{2}(\varepsilon)$ is presented in Fig. 4 at three values of median drop diameter $D_{0}[\mathrm{~mm}]$, namely: 1.1 (dashed violet curve), 1.3 (dotted blue curve), and 1.5 (solid red curve). Note that $D_{0}$ is strongly correlated with the rain rate $R$ [8]. Calculations show that $D_{0}=1.1 \mathrm{~mm}$ corresponds to $R \approx 3.2 \mathrm{~mm} / \mathrm{h}$; $D_{0}=1.3 \mathrm{~mm} \rightarrow R \approx 7 \mathrm{~mm} / \mathrm{h} ; D_{0}=1.5 \mathrm{~mm} \rightarrow R \approx 13.7 \mathrm{~mm} / \mathrm{h}$.

The calculations were done at $L_{u l}=30 \mathrm{~m} ; L_{o}=1000 \mathrm{~m}$.

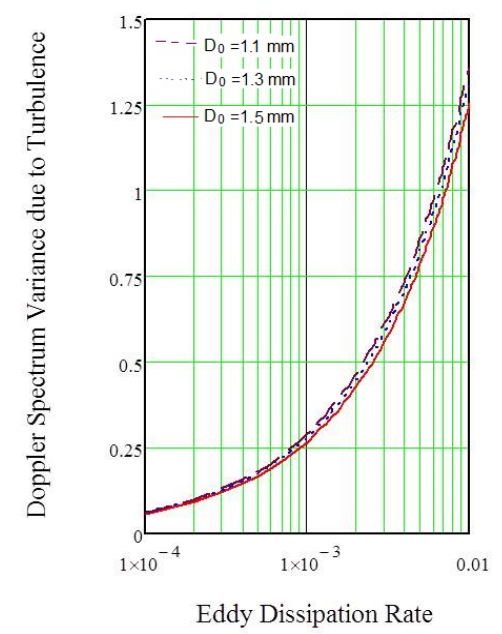

Fig. 4. Doppler spectrum variance $\left[\mathrm{m}^{2} / \mathrm{s}^{2}\right]$ induced by turbulence versus EDR $\left[\mathrm{m}^{2} \mathrm{~s}^{-3}\right]$ at different $D_{0}$. EDR is shown in log scale and includes all levels of turbulence intencity since negligible to strong.

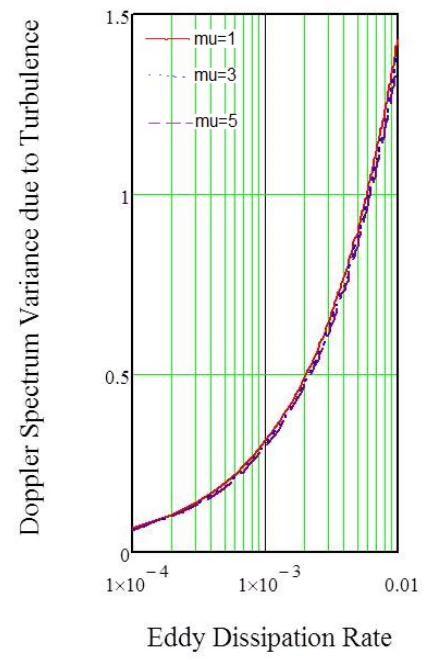

Fig. 5. Doppler spectrum variance $\left[\mathrm{m}^{2} / \mathrm{s}^{2}\right]$ induced by turbulence versus EDR $\left[\mathrm{m}^{2} \mathrm{~s}^{-3}\right]$ at different $\mu$. 
It is worth to mention that the relationship $D_{0} \leftrightarrow R$ is valid at practically any value of $\mu$. Calculations of the variance (18) versus $\operatorname{EDR} \sigma_{v, T}^{2}(\varepsilon)$ done at constant $D_{0}=1.5$ and three values of $\mu$ (DSD shape parameter) show that relation $\sigma_{v, T}^{2} \leftrightarrow \varepsilon$ also quite weakly depends on $\mu$; it is even possible to say that reasonable values of $\mu$ does not influence onto the character of $\sigma_{v, T}^{2}(\varepsilon)$. This is confirmed by Fig. 5, where three curves at different $\mu$ are presented: $\mu=1$ (solid red curve), $\mu=3$ (dotted blue curve), and $\mu=5$ (dashed violet curve). All curves practically coincide.

Dependence of a turbulent part of the DSW as function of $D_{0}$ is shown in Fig. 6 at three values of turbulence intensity $\mathcal{E}$ : $0.001 ; 0.005 ; 0.01\left[\mathrm{~m}^{2} \mathrm{~s}^{-3}\right]$.

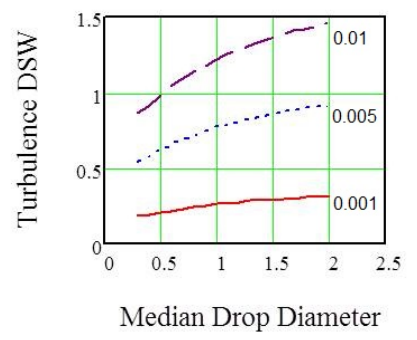

Fig. 6. Doppler spectrum variance $\left[\mathrm{m}^{2} / \mathrm{s}^{2}\right]$ induced by turbulence versus median drop diameter at three values of EDR $\left[\mathrm{m}^{2} \mathrm{~s}^{-3}\right]$.

One can see that increasing $D_{0}$ and hence relative number of large drops increases slightly the turbulence DSW. This effect manifests more, if the turbulence is stronger.

\section{COMPARISON OF DFV AND TURBULENCE CONTRIBUTIONS TO THE DOPPLER SPECTRUM WIDTH}

Now having the models of both main components of the Doppler spectrum variance we can estimate the total variance as a sum of gravitational (DFV) and turbulent contributions according to (1). The results of calculations are collected in Figs $7-10$ at different turbulence intensity from very strong to light turbulence.

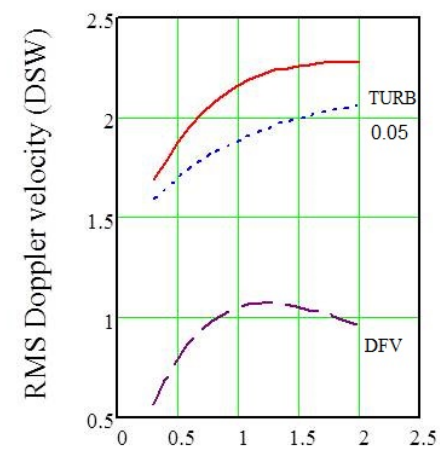

Median Drop Diameter

Fig. 7. Doppler spectrum width $[\mathrm{m} / \mathrm{s}]$ induced by turbulence (blue dots), DFV dispersion (violet dashes), and total DSW versus $\mathrm{D}_{0}$ at very strong turbulence $\left(\mathrm{EDR}=0.05 \mathrm{~m}^{2} \mathrm{~s}^{-3}\right)$.

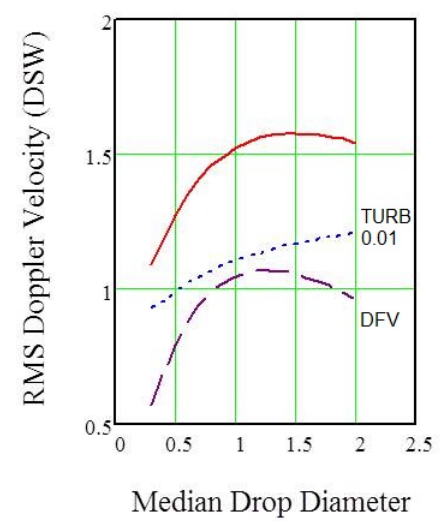

Fig. 8. Doppler spectrum width $[\mathrm{m} / \mathrm{s}]$ induced by turbulence (blue dots), DFV dispersion (violet dashes), and total DSW versus $\mathrm{D}_{0}$ at strong turbulence $\left(\mathrm{EDR}=0.01 \mathrm{~m}^{2} \mathrm{~s}^{-3}\right)$.

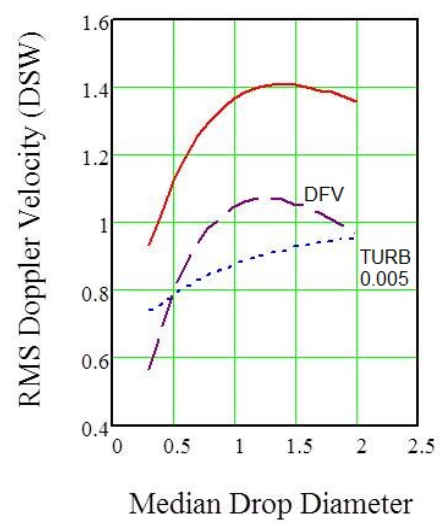

Fig. 9. Doppler spectrum width $[\mathrm{m} / \mathrm{s}]$ induced by turbulence (blue dots), DFV dispersion (violet dashes), and total DSW versus $\mathrm{D}_{0}$ at moderate turbulence $\left(\mathrm{EDR}=0.005 \mathrm{~m}^{2} \mathrm{~s}^{-3}\right)$.

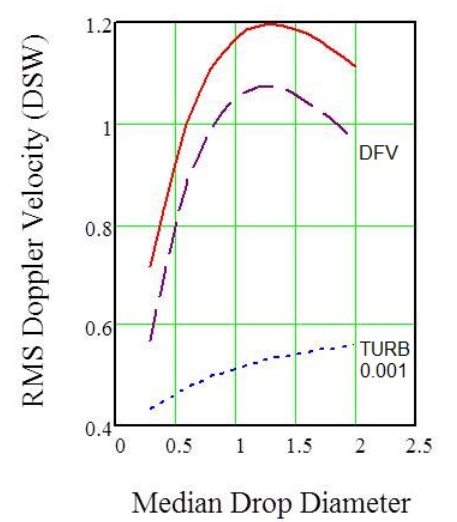

Fig. 10. Doppler spectrum width $[\mathrm{m} / \mathrm{s}]$ induced by turbulence (blue dots), DFV dispersion (violet dashes), and total DSW versus $D_{0}$ at light turbulence $\left(\mathrm{EDR}=0.001 \mathrm{~m}^{2} \mathrm{~s}^{-3}\right)$.

In these pictures red solid curves are total DSW $\sigma_{v}$ in accordance with (1), that is,

$$
\sigma_{v}=\sqrt{\sigma_{v, f}^{2}+\sigma_{v, T}^{2}}
$$


blue dotted curves represent a part of the Doppler spectrum width induced by turbulence $\sigma_{v, T}$ as is defined by (18), and violet dashed curves are $\sigma_{v, f}=W_{d n}$ described by (11). Analysis of these plots clearly shows that values of $\sigma_{v, T}$ and $\sigma_{v, f}$ can be of the same order. While in case of strong turbulence the main contribution to DSW comes from $\sigma_{v, T}$ (Fig. 7) and in case of light turbulence it comes from $\sigma_{v, f}$ (Fig. 10), the intermediate situations (Fig. 8 and Fig. 9) show really similar values of the two. That is why the task of separation turbulent and gravitational components based on DSW measurement can be rather difficult even in case of raindrops as radar scatterers. In case of other types of hydrometeors as the scatterers [13] the task can be more complicated.

\section{POSSIBLE SIGNAL PROCESSING ALGORITHMS TO RETRIEVE INFORMATION ON TURBULENCE INTENSITY}

The idea to subtract the estimate of $\sigma_{v, f}^{2}$ from measured value of $\sigma_{v}^{2}$ to get the desired estimate of $\sigma_{v, T}^{2}$ is rather obvious. The question is how to estimate the value $\sigma_{v, f}^{2}$. In [6] it was implemented by rather simple way, using measurements of radar reflectivity $Z=f\left(P_{r}\right)$ with calibrated radar based on radar equation with conversion $Z$ to the rain rate $R$ and then to median drop diameter, which is necessary to retrieve $\sigma_{v, f}^{2}$ using (11) and other related formulas. There are a lot of works dedicated to retrieving parameters of rain microstructure, that is, parameters $D_{0}$ and $\mu$ from polarimetric radar data [9], [14], [15]. The details of estimating DFV contribution are off the scope of this paper.

An important problem is to provide acceptable accuracy of measurement and particularly $\sigma_{v, f}^{2}$ estimate. And first of all it is necessary to establish the requirements to the accuracy of such estimation. On the one hand it seems that even not very accurate estimation of gravitational part of DSW may improve the final estimate of the turbulent component because otherwise DSW definitely overestimates the unknown quantity of EDR. On the other hand the inaccurate estimate of $\sigma_{v, f}^{2}$ can occur even more than measured total $\sigma_{v}^{2}$ that leads to completely wrong results. Such possibility should be taken into account in the signal processing algorithm.

Thus, the accuracy of EDR retrieval from DSW is limited by the following sources of errors: DSW estimation, DFV estimation, contributions that are not included in (1) [8], inaccuracy of conversion $\varepsilon \approx f\left(\sigma_{v, T}\right)$, etc. It should be studied analytically and by computer simulation. The developed models can be used as the basis for further statistical simulation aimed to substantiate the requirements to allowable errors of DFV estimation to support desired accuracy of measuring turbulence of given intensity.

\section{CONCLUSION AND FUTURE WORK}

The developed models are necessary for EDR retrieval based on the DSW, using correction algorithm with adaptive estimation of a gravity contribution to the variance of Doppler spectrum. The models will be applied to analyze and substantiate allowable errors of estimating drop fall velocity contribution to the measured Doppler spectrum. They will be used for the development of signal processing algorithms and further comparison with in-situ measurements [10].

\section{ACKNOWLEDGMENT}

This work was supported by the EU FP7 UFO project.

\section{REFERENCES}

[1] A.S. Frisch and R.G. Strauch, "Doppler Radar Measurements of Turbulent Kinetic Energy Dissipation Rates in a Northeastern Colorado Convective Storm,” J. Appl. Meteorology, 15, 1976, pp. 1012-1017.

[2] W.K. Hocking, "Observation and measurement of turbulence in the middle atmosphere with a VHF radar," J. Atmos and Terrestrial Physics, Vol 48, No. 7, 1986, pp.6 55-670.

[3] M.D. Shupe, I.M. Brooks, and G. Canut, "Evaluation of turbulent dissipation rate retrievals from Doppler Cloud Radar," Atmospheric Measurement Technique, 5, 2012, pp. 1375-1385.

[4] A.K. Bohne, "Radar detection of turbulence severity in precipitation environments," J. Atmos. Sci. 39, 1982, pp. 1819-1837.

[5] F.J. Yanovsky, I.G. Prokopenko, K.I. Prokopenko, H.W.J. Russchenberg, and L.P. Ligthart, "Radar Estimation of Turbulence Eddy Dissipation Rate in Rain,” Proc. IEEE IGARSS, Toronto, Canada, 2002, Vol. 1, 2002, pp. 63-65.

[6] F.J. Yanovsky, H.W.J. Russchenberg, and C.M.H. Unal, "Retrieval of Information About Turbulence in Rain by Using Doppler-Polarimetric Radar," IEEE Trans. MTT, Vol. 53, No. 2, Feb 2005, pp. 444-450.

[7] N.K. Vinnichenko, N.Z. Pinus, S.M. Shmeter, and G.N. Shur, Turbulence in the free atmosphere, Gidrometeoizdat, Leningrad, 1968, 336 pp. (in Russian)

[8] R. J. Doviak and D. S. Zrnic, Doppler Radar and Weather Observations, 2nd ed. San Diego, CA: Academic, 1993.

[9] V.N. Bringi and V. Chandrasekar, Polarimetric Doppler Weather Radar. Cambridge, U.K.: Cambridge Univ. Press, 2002.

[10] F.J. Yanovsky, A.C.P. Oude Nijhuis, O.A. Krasnov, C.M.H. Unal, H.W.J. Russchenberg, and A. Yarovoy, "Turbulence Intensity Estimation Using Advanced Radar Methods," Accepted paper to EuRAD-2015, Paris, 2015.

[11] F. Yanovsky, Inferring microstructure and turbulence properties in rain through observations and simulations of signal spectra measured with Doppler-polarimetric radars. Book Chapter in: Polarimetric Detection, Characterization, and Remote Sensing, Springer, 2011, pp. 501-545.

[12] F.J. Yanovsky, "Simulation study of $10 \mathrm{GHz}$ radar backscattering from clouds and solution of the inverse problem of atmospheric turbulence measurements," Proceedings of the Conference "Computation in Electromagnetics", IEE No. 420, 1996, pp. 188-193.

[13] F.J. Yanovsky, I.M. Braun, "Models of Scattering on Hailstones in Xband," Proceedings of the European Radar Conference EuRAD 2004, Amsterdam, 2004, pp. 229-232.

[14] V.N. Bringi, V. Chandrasekar, J. Hubbert, E. Gorgucci, W L Randeu and M. Schönhuber, "Raindrop size distribution in different climatic regimes from disdrometer and dual-polarized radar analysis", J. Atmos. Sci., 2003, 60, pp. 354-365.

[15] D.N. Glushko and F.J. Yanovsky, "Analysis of differential Doppler velocity for remote sensing of clouds and precipitation with dualpolarization S-band radar," Int. J. Microwave and Wireless Technologies, 2010, Vol. 2, issue 3-4, pp. 391-398. 